\title{
Tetanus antigen modulates the gene expression profile of aluminium phosphate adjuvant in spleen lymphocytes in vivo
}

K Regnström

EGE Ragnarsson

N Rydell

I Sjöholm

P Arthursson

Correction to: The Pharmacogenomics Journal (2002) 2: 57-64. doi:10.1038/sj.tpj.6500080

The Pharmacogenomics Journal (2002) 2, 341. doi:10.1038/sj.tpj.6500141

Supplementary material for Figure 4 of this article should have been published online alongside the paper at the rime of publication. We regret that this information was not loaded at the time but would point out that it has now been added to the online article.

Please access the article through The Pharmacogenomics Journal website which can be found at: www.nature.com/tpj. We apologise for any inconvenience this may have caused. 